\title{
Análisis estructural numérico del modelo a escala 1:10 del cascarón no-canónico "Yasunî" generado sobre la base de la arquitectura biónica
}

\section{Numerical structural analysis of the 1:10 scale model of the "Yasuni" non-canonical shell generated on the basis of bionic architecture}

\author{
David Cajamarca Zúñiga ${ }^{1 *}$ y Olga Alyoshina ${ }^{2}$ \\ Universidad Católica de Cuenca ${ }^{1}$ \\ RUDN University ${ }^{2}$ \\ *cajamarca.zuniga@gmail.com \\ DOI: https://doi.org/10.26871/killkana_tecnica.v3i1.414
}

\begin{abstract}
Resumen
Los autores continúan el estudio el estado tenso-deformacional de una nueva forma no-canónica de cascarón estructural. El estudio se realiza sobre un modelo a escala 1:10 de la envolvente "Yasunín", mediante un sistema de cálculo basado en el Análisis de Elementos Finitos de estructuras (AEF) con el empleo de una malla triangular planteada a partir de la concepción arquitectónica, que fusiona armónicamente en una sola estructura las formas características de la flora y fauna de la Amazonía ecuatoriana. La envolvente se analiza en un modelo de hormigón armado de diferentes resistencias $\left(f^{\prime} c=18,5 M P a, f^{\prime} c=22 M P a\right.$ y $\left.f^{\prime} c=25,5 M P a\right)$, con espesores que varían desde $\mathrm{t}=5 \mathrm{~cm}$ hasta $\mathrm{t}=10 \mathrm{~cm}$ con incrementos de $1 \mathrm{~cm}$, sin vigas de borde y bajo la acción del peso propio. En este artículo se presentan los resultados de la investigación del estado tenso-deformacional del modelo a escala del cascarón "Yasuní" y se observa que a mayor espesor del cascarón las magnitudes de las tensiones normales decrecen, mientras que los valores de las cortantes y momentos flexores se incrementan. Seguir con la investigación de esta envolvente, considerando la modificación de diversos parámetros geométricos, de carga, materiales, condiciones de borde y otros.
\end{abstract}

Palabras clave: Análisis de Elementos Finitos, arquitectura biónica, cascarón no-canónico, estado tensodeformacional, estructura de hormigón armado, malla triangular de elementos finitos.

\begin{abstract}
In this paper, the authors continue with the study of the tension-deformational state of a new non-canonical form of structural shell. The study is carried out on a 1:10 scale model of the "Yasuni" lenvelope, by means of a calculation system based on the Finite Element Analysis of structures (FEA), using a triangular mesh which is based on the architectural conception, that harmonically fuses, in a single structure, the characteristic forms of the flora and fauna in the Ecuadorian Amazonie The envelope is analyzed in a reinforced concrete model of different compression resistance $\left(f^{\prime} c=18.5 M P a\right.$, $f^{\prime} c=22 \mathrm{MPa}$ y $f^{\prime} c=25.5 \mathrm{MPa}$ ) which thicknesses ranges from $t=5 \mathrm{~cm}$ to $t=10 \mathrm{~cm}$ in $1 \mathrm{~cm}$ increments, without edge beams and under the action of its own weight. This article presents the research results on the tension-deformational state of the scale model of the "Yasuni" envelope and shows that when the shell thickness is greater, the magnitudes of the normal tension decreases, while the values of the shears and flexor moments increase. The authors will continue to investigate this envelope, considering the modification of various geometric parameters, loads, materials, edge conditions and others.
\end{abstract}

Key words: Finite Element Analysis, bionic architecture, non-canonic shell, tension-deformational state, reinforced concrete structure, finite element triangular mesh.

\section{INTRODUCCIÓN}

Es difícil concebir la arquitectura y la construcción sin considerar las estructuras envolventes de pared delgada de grandes luces, las mismas que tienen diversas aplicaciones y usos [1]. Algunos autores señalan que la utilización de estructuras de grandes luces, ha perdido su popularidad en comparación con la época pico de su construcción en los 
años 1950-1960 [2]. Sin embargo, en estos tiempos se tiene una nueva generación de arquitectos e ingenieros, quienes demuestran su interés por el diseño de estructuras de cubierta de grandes luces, además de la aparición de nuevas formas en la arquitectura de las estructuras espaciales. Por ejemplo, en el departamento de resistencia de materiales y estructuras de la RUDN University de Moscú, se ha elaborado la Enciclopedia de Superficies Analíticas [3], que contiene más de 500 tipologías, de las cuales la mayoría pueden ser asumidas como una base arquitectónica para el diseño de estructuras espaciales de pared delgada.

Por lo tanto, las nuevas exigencias de los arquitectos, los nuevos alcances de la modelación numérica de superficies y la introducción de nuevos grupos de superficies estructurales con determinadas propiedades, vuelven inevitable la aparición de nuevas formas y tipos de estructuras envolventes o cascarones [3], y los métodos de la arquitectura biónica amplían los límites de las alternativas para resolver esta tarea [4].

En julio de 2017 el autor del presente trabajo se percató que el joven arquitecto José Pesántez, de la Universidad Católica de Cuenca, basado en los criterios de la arquitectura biónica, había planteado una nueva forma no-canónica de cascarón denominado "Yasuní" (Figura 1) concebido para el Centro de Interpretación de la Naturaleza en el Parque Nacional (reserva de la biosfera) de Yasuní en Ecuador, esta estructura generó la discusión de los especialistas estructurales, razón por la que es objeto de investigación de su estado tenso-deformacional

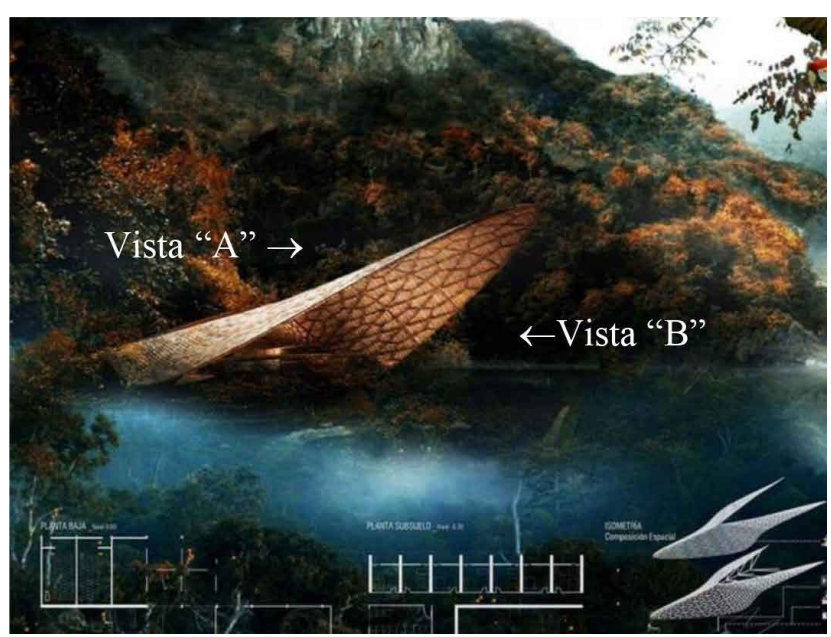

Fig. 1. Cascarón "Yasunî"

Fuente: Arq. José Pesántez
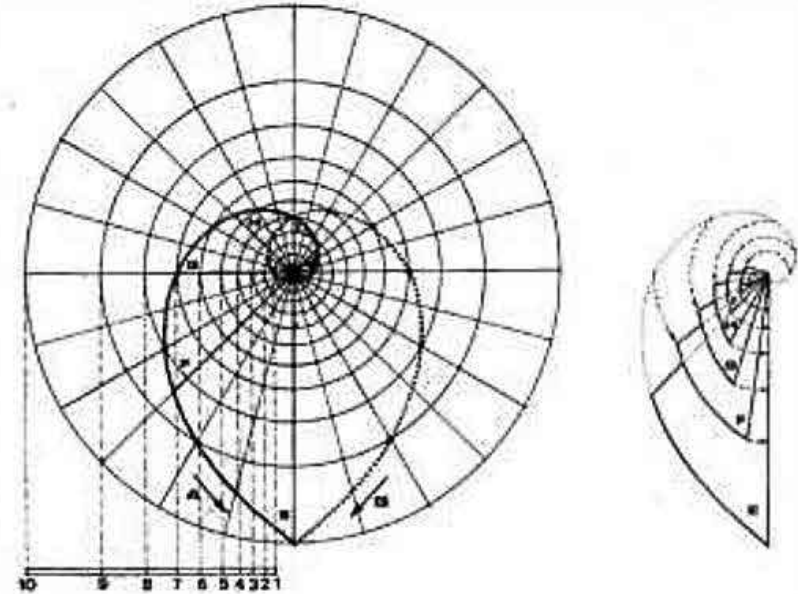

Fig. 2. Diagrama de una hoja de margarita

Fuente: [5]

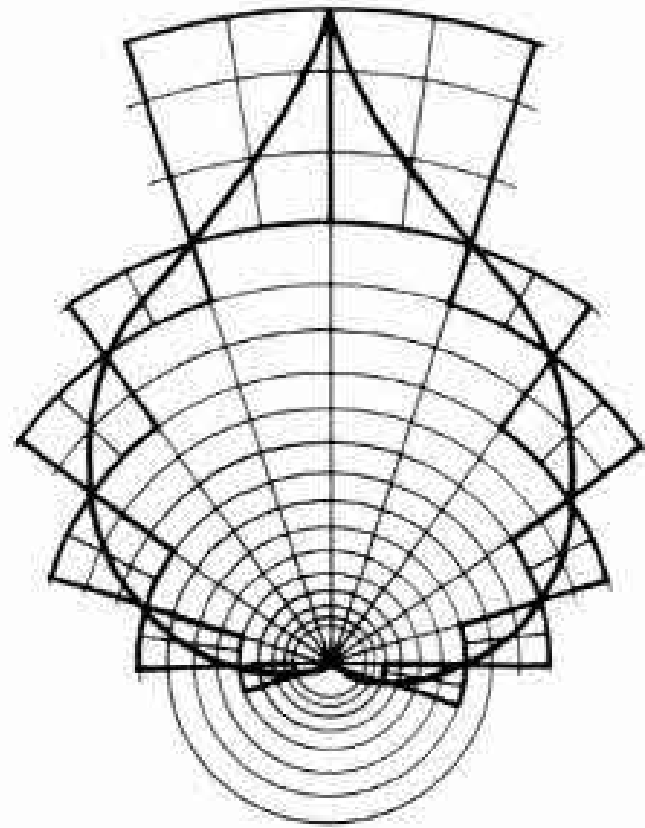

Fig. 3. Diagrama de una hoja de Lilia

Fuente: [5]

La concepción arquitectónica de la forma del cascarón "Yasuní" plantea que la morfología de la envolvente imite la forma de las hojas que se revelan dispersas en el territorio, las mismas que se acomodan sobre el relieve topográfico sin modificar la permeabilidad natural que presenta la superficie tropical donde se emplazaría la estructura. La disposición y el perfil del trazo de la hoja han sido concebidos por las relaciones entre los patrones de crecimiento, es decir, que el conjunto de elementos que componen las hojas (nervaduras, limbo y ramas) sean entendidos análogamente como elementos de la estructura (entramado de barras, capas de recubrimiento y elementos de apoyo). La concepción arquitectónica plantea que las 
resultantes generadas de los radios proporcionales (Figura 2, Figura 3), creen una armonía surgida de la capacidad de aunar las partes de la hoja, conservando cada una de ellas su identidad, para integrarse en un elemento monolítico que ordene y organice el espacio, y eleve de esta manera la cotidianidad de habitar al nivel del arte y en equilibrio con la naturaleza [5].

En este artículo se presentan los resultados de la continuación de la investigación del estado tenso-deformacional (ETD) de esta nueva forma no-canónica de envolvente estructural, que fue iniciada por los autores y que se presentó en el artículo [6]. Para este análisis se plantea la estructura propuesta por el arquitecto (Figura 1), como una estructura de pared delgada de hormigón monolítico en una escala 8 veces menor. Este modelo a escala respeta íntegramente la forma geométrica original y permite estudiar y conocer su comportamiento estructural con el propósito de evidenciar su comportamiento y recomendar la elaboración del modelo físico para comprobar los datos del modelo matemático.

Se ha logrado evidenciar que el estado tensodeformacional del cascarón varía de forma considerable en función de las condiciones de borde, como de la presencia o ausencia de elementos de contorno y del tipo de carga (estática, de viento o sísmica). Para la determinación de las reacciones en la estructura bajo la acción de las cargas y la definición de los parámetros geométricos característicos del cascarón propuesto se utiliza el método de elementos finitos [7]. Los cálculos se realizan en el complejo computacional SCAD, el cual permite encontrar el patrón de trabajo de la estructura y considerar todas las particularidades de la solución constructiva [8].

En la Figura 4 y Figura 5 se indican las vistas generales del modelo matemático de cálculo, con la aproximación de la superficie media de la envolvente a un conjunto de elementos finitos triangulares planos [9].

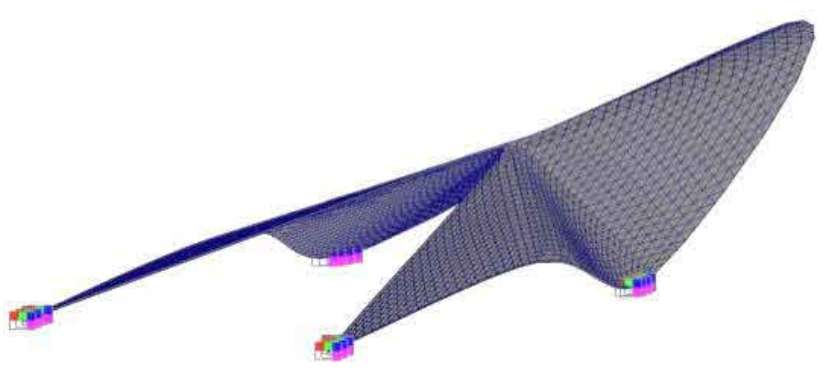

Fig. 4. Modelo de cálculo. Vista A

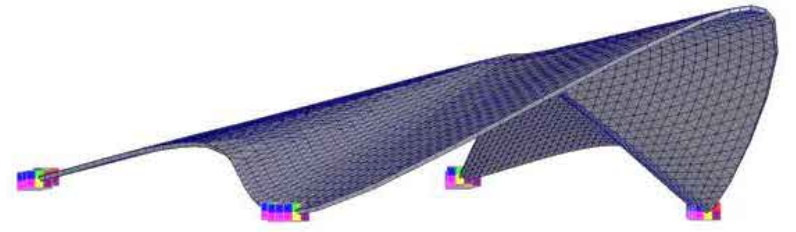

Fig. 5. Modelo de cálculo. Vista B

El modelo de cálculo de la envolvente prevé cuatro puntos de apoyos empotrados. Las dimensiones geométricas generales del modelo de cálculo a escala 1:10 son de $17.4 \times 4.8 \mathrm{~m}$ en el plano y una flecha de elevación de 2.2 $\mathrm{m}$, las dimensiones de los lados de los elementos finitos triangulares varían entre $0.1 \mathrm{~m}$ y $0.4 \mathrm{~m}$, y se cuenta con 3438 elementos triangulares y 1813 nudos.

En esta investigación, el análisis de la envolvente se realiza con hormigón armado de diferentes resistencias a la compresión $f^{\prime} c=18,5 M P a, f^{\prime} c=22 M P a$ y $f^{\prime} c=$ 25,5MPa, y módulos de elasticidad E respectivamente de $30000 \mathrm{MPa}, 32500 \mathrm{MPa}$ y $34500 \mathrm{MPa}$ (Scientific Research, 2004), con un espesor que varía de $\mathrm{t}=5 \mathrm{~cm}$ hasta $\mathrm{t}=10$ $\mathrm{cm}$ con incrementos de $1 \mathrm{~cm}$, sin vigas de borde y bajo la acción solo del peso propio como carga gravitacional uniformemente distribuida.
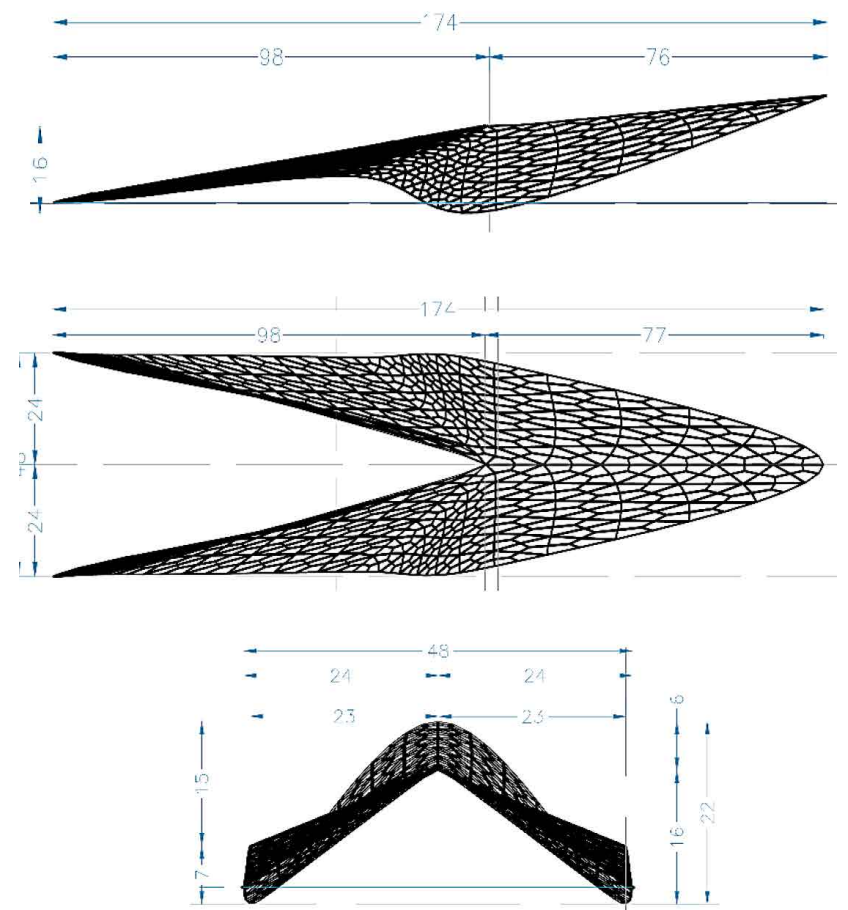

Fig. 6. Geometría. Vista lateral, en planta y frontal del modelo

En las siguientes figuras se indican algunos resultados del cálculo y modelación matemática de la envolvente de 5 cm de espesor, con hormigón de $f^{\prime} c=18,5 M P a$. 


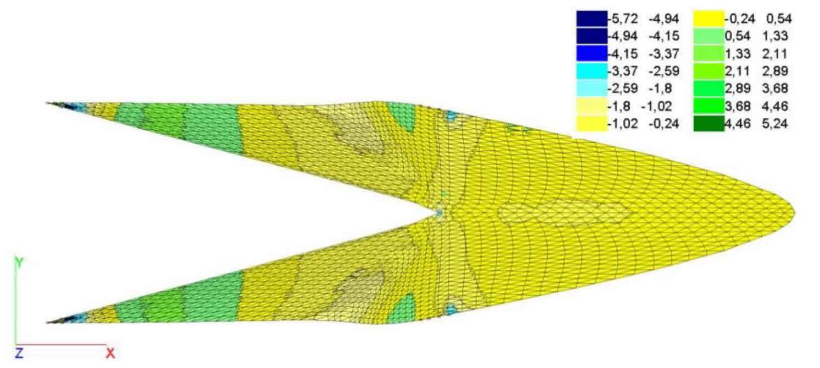

Fig. 7. Momento flexor $M_{x}(\mathrm{kN}-\mathrm{m} / \mathrm{m})$

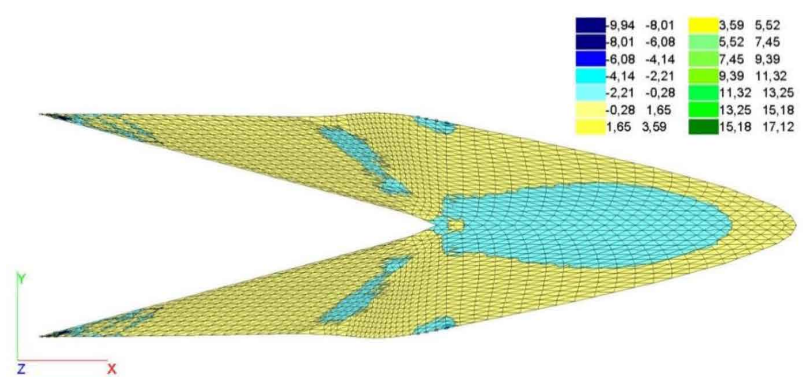

Fig. 8. Momentos flexores $M_{y}(\mathrm{kN}-\mathrm{m} / \mathrm{m})$

El análisis del carácter de la distribución de los isocampos de momentos flectores $M_{x}$ (Figura 7) y $M_{y}$ (Figura 8) sugiere que el cascarón trabaja según el esquema estructural de una barra en la dirección x. La magnitud máxima del momento flexor $M_{x}$ en el vano entre los apoyos es de 2.1 $\mathrm{kN}-\mathrm{m} / \mathrm{m}$, y en la parte del voladizo la magnitud oscila entre $-0.24 \mathrm{kN}-\mathrm{m} / \mathrm{m}$ y $0.54 \mathrm{kN}-\mathrm{m} / \mathrm{m}$. Se puede observar un incremento considerable de los momentos flectores negativos en las zonas de los apoyos posteriores del cascarón debido a las fuerzas de tracción generadas por los conectores. Estos esfuerzos, para la geometría propuesta, podrían ser asumidos por vigas de borde o una variación en la geometría del cascarón, lo cual se continúa investigando y los resultados serán presentados en un siguiente artículo.

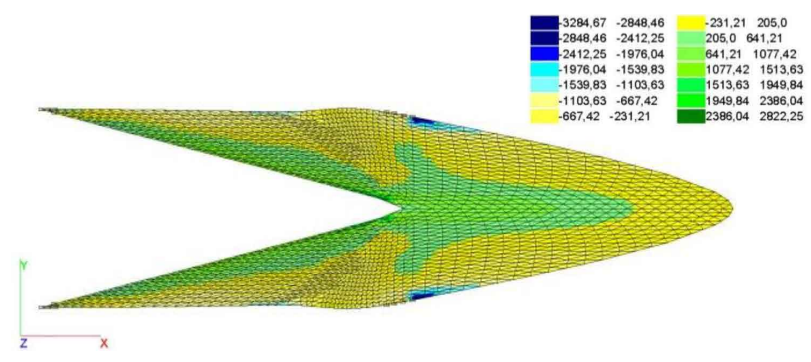

Fig. 9. Tensiones normales $N_{x}(\mathrm{kN} / \mathrm{m} 2)$

En la Figura 9 se observan los isocampos de tensiones normales $\mathrm{Nx}$, los cuales evidencian el carácter del trabajo del cascarón que presenta un comportamiento de viga en voladizo en la dirección x. Además, se puede observar que las normales negativas que se desarrollan son producto de la geometría del cascarón y son en su totalidad asumidas por el material.

En la Figura 10 se indican los isocampos de desplazamientos verticales, en donde se observa que el voladizo se desplaza hasta $4.6 \mathrm{~mm}$ y los máximos desplazamientos surgen entre los apoyos, alcanzando los $13 \mathrm{~mm}$.

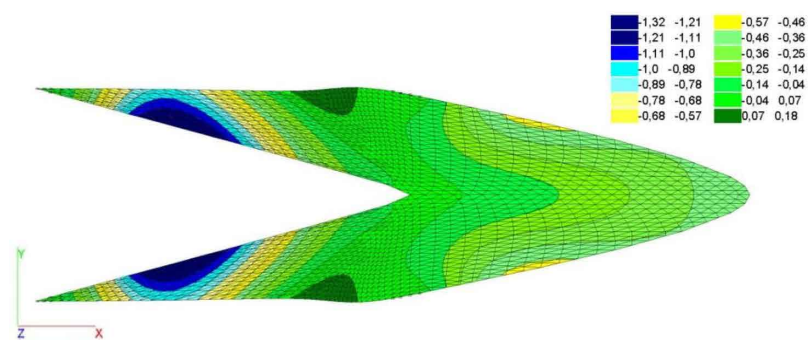

Fig. 10. Desplazamientos verticales $(\mathrm{cm})$

En la Tabla I se presentan los desplazamientos verticales máximos debido a la carga uniformemente distribuida del peso propio del cascarón, en donde se puede observar que, para los diferentes espesores de la estructura considerados para esta investigación, el carácter general de distribución de los isocampos de desplazamientos es similar.

TABLA I. Desplazamientos verticales máximos por la acción del peso propio

\begin{tabular}{|c|c|c|c|}
\hline \multirow{2}{*}{$\begin{array}{c}\text { Espesor } \\
\mathrm{t}(\mathrm{cm})\end{array}$} & \multicolumn{3}{|c|}{ MAX DESPLAZAMIENTO VERTICAL $(\mathrm{cm})}$, \\
& SEGÚN RESISTENCIA DEL HORMIGÓN $f^{\prime} c(\mathrm{MPa})$ \\
\cline { 2 - 4 } & $\mathbf{1 8 . 5} \mathbf{~ M P a}$ & $\mathbf{2 2} \mathbf{~ M P a}$ & $\mathbf{2 5 . 5} \mathbf{~ M P a}$ \\
\hline 5 & -13.2 & -12.2 & -11.5 \\
\hline 6 & -9.9 & -9.1 & -8.6 \\
\hline 7 & -7.7 & -7.1 & -6.7 \\
\hline 8 & -6.2 & -5.7 & -5.3 \\
\hline 9 & -5.1 & -4.7 & -4.4 \\
\hline 10 & -4.2 & -3.9 & -3.7 \\
\hline
\end{tabular}

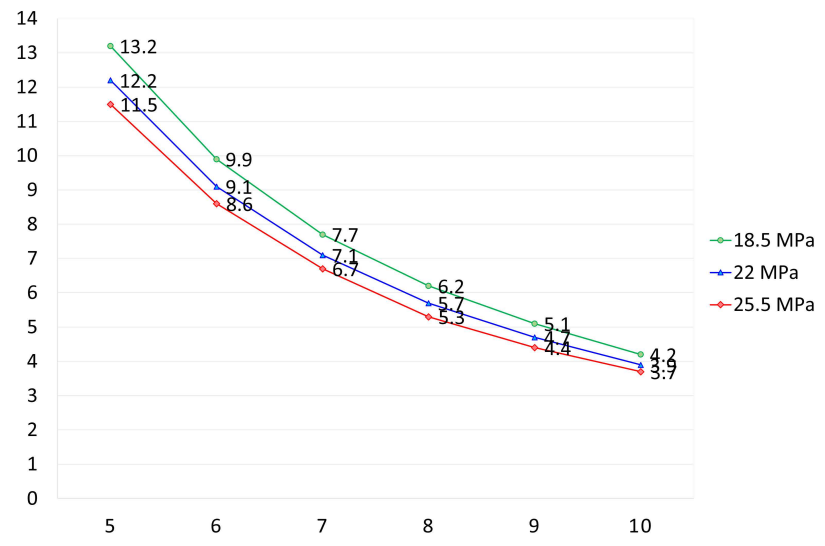

Fig. 11. Relación Desplazamiento vertical - Espesor del cascarón, según la resistencia del hormigón 


\section{CONCLUSIONES}

En el análisis de las alternativas propuestas se observa que:

- A mayor espesor del cascarón existe una menor influencia de la resistencia del hormigón sobre los desplazamientos verticales.

- Las magnitudes de las tensiones normales $\left(N_{x}, N_{y}\right)$, esfuerzos transversales $\left(Q_{x}, Q_{y}\right)$ y momentos flexores $\left(M_{x}, M_{y}\right)$ son iguales sin importar la resistencia del hormigón.

- Para una determinada resistencia del hormigón, cuando se incrementa el espesor del cascarón las tensiones normales $\left(N_{x}, N_{y}\right)$ decrecen, mientras que los valores de las fuerzas transversales $\left(Q_{x}, Q_{y}\right)$ y de los momentos flexores $\left(M_{x}, M_{y}\right)$ se incrementan, lo que ratifica el carácter del trabajo del cascarón como una estructura laminar de pared delgada y corrobora que este tipo de estructuras tienen un comportamiento estructural racional mientras menor sea su espesor.

- Se estima que la utilización de hormigones de altas prestaciones sería favorable para elaborar cascarones de menores espesores ya que existiría una mayor influencia sobre el control de deflexiones y mejor comportamiento ante las solicitaciones desarrolladas.

En este artículo se ha continuado la investigación del estado tenso-deformacional del cascarón "YASUNI" presentada en [6]. Esta investigación continuará en curso y se estudiará el estado tenso-deformacional de este cascarón con la modificación de diferentes parámetros geométricos, materiales y condiciones de frontera y la introducción de elementos de borde o rigidizadores transversales sobre un modelo numérico a escala real.

\section{REFERENCIAS}

[1] S. N. Krivoshapko and I. A. Mamieva, "Outstanding spatial structures of the last 20 years," Mounting and special works in construction, no. 12, pp. 8-14, 2012.

[2] S. N. Krivoshapko and I. A. Mamieva, Analytical surfaces in the architecture of buildings and structures. Moscow, Russia: LIBROKOM, 2011.
[3] S. N. Krivoshapko and V. N. Ivanov, Encyclopedia of Analytical Surfaces. Switzerland: Springer International Publishing Switzerland, 2015.

[4] Y. S. Lebedev, V. I. Rabinovich, E. D. Polozhay, and et Al., Architectural Bionics. Moscow, Russia: Stroyizdat, 1990.

[5] G. Doczi, El poder de los límites: proporciones armónicas en la naturaleza, el arte y la arquitectura. Buenos Aires: Troquel, 2006.

[6] O. O. Alyoshina and D. Cajamarca Zuniga, "Stressstrain state investigation of the "Yasuni"shell by bionic architecture methods based on numerical modeling," STRUCTURAL MECHANICS OF ENGINEERING CONSTRUCTIONS AND BUILDINGS, vol. 14, no. 4, pp. 273-277, 2018.

[7] L. A. Rozin and L. A. Gordon, "The finite element method in the theory of plates and shells," Izvestiya VNIIG, no. 95, 1971.

[8] V. S. Karpilovsky, E. Z. Kriksunov, A. A. Malyarenko, A. V. Perelmuter, and S. Y. Fialko, SCAD Office. Version 21. Computing complex SCAD ++. Moscow: SCAD Soft, 2015.

[9] L. J. Segerlind, Applied Finite Element Analysis. Michigan State University, 1976.

Recibido: 24 de enero de 2019

Aceptado: 15 de marzo de 2019 
